August 4-7, 2013, Portland, Oregon, USA

DETC2013-13252

\title{
CONSIDERATION OF ASSISTIVE DEVICES IN THE GAIT ANALYSIS OF SPINAL CORD-INJURED SUBJECTS
}

\author{
Urbano Lugrís* \\ Department of Industrial Engineering II \\ University of La Coruña \\ Ferrol, 15403, Spain \\ Email: ulugris@udc.es
}

\author{
Alberto Luaces \\ Department of Industrial Engineering II \\ University of La Coruña \\ Ferrol, 15403, Spain \\ Email: aluaces@udc.es
}

\author{
Jairo Carlín \\ Department of Industrial Engineering II \\ University of La Coruña \\ Ferrol, 15403, Spain \\ Email: jairo.carlin@udc.es \\ Javier Cuadrado \\ Department of Industrial Engineering II \\ University of La Coruña \\ Ferrol, 15403, Spain \\ Email: javicuad@cdf.udc.es
}

\begin{abstract}
The gait of spinal cord-injured subjects can be improved by means of properly designed active orthoses. Since the gait pattern varies greatly among different patients, the orthoses and their corresponding controllers must be carefully customized, and the joint motor torques obtained from inverse dynamic analysis constitute a useful input for this task.

Nowadays, the analysis of standard gait by using motion capture data is a mature discipline. However, the problem becomes more complex in the case of spinal cord-injured subjects wearing active orthoses: in addition to the presence of the orthoses, these patients always require the help of supplementary assistive devices, such as crutches, in order to stabilize their gait.

In this work, the gait analysis of a subject walking with the aid of crutches and active knee-ankle-foot orthoses is performed, by introducing the assistive devices both at the experimental and computational levels. The required sensors and actuators are incorporated to the system, and the measurements are used to solve the inverse dynamics problem in order to calculate the joint motor torques produced by the subject during gait.
\end{abstract}

\footnotetext{
*Address all correspondence to this author.
}

\section{INTRODUCTION}

Many spinal cord-injured (SCI) subjects with no ability to control their knee and ankle joints can walk with the help of assistive devices, such as crutches and orthoses. The knee-anklefoot orthoses (KAFOs) commonly used by these patients lock the knee joint flexion-extension in order to bear their weight, thus leading to an energetically inefficient gait. There are several orthosis models in the market which allow to improve the gait by unlocking the knee flexion during leg swing, but they do not provide the necessary torque to perform the flexion-extension motion, and in many cases they require the patient to lock/unlock the knee manually at each step, by means of a remote control device. The development of an active KAFO $[1,2]$ with an actively controlled knee joint can greatly reduce the metabolic cost, which would encourage the patients to walk instead of using wheelchairs, thus improving their rehabilitation.

Since the gait pattern varies greatly among different patients, the control strategy of the KAFO must be carefully customized to fit the patient's needs. In order to design and evaluate the performance of the orthosis control, inverse dynamic (ID) analysis of the gait is a very useful tool. The analysis requires the ex- 
istence of an experimental setup for measuring gait data, which is fed into a computational model that calculates the joint motor torques. This paper addresses the introduction of assistive devices, i.e. orthoses and crutches, into an existing gait analysis system. This presents several problems like the modeling of the orthosis and the presence of multiple ground contacts due to the use of crutches [3]. There exist previous works in the literature addressing crutch-assisted gait $[4,5]$, but they are focused on the efforts produced at the upper-body joints.

The active orthoses under development [6] have actuated knee joints, including a motor and a mechanical locking device, and passive (Klenzack) ankle joints. The controller uses the knee and ankle angles as inputs, along with the information from plantar pressure sensors. Each orthosis link is introduced in the model as a rigid body, firmly attached to the corresponding body segment. The ankle Klenzack springs are straightforwardly considered, while the torques provided by the knee motors are taken into account by introducing the control algorithm into the model.

In order to perform the analysis, a pair of standard crutches have been instrumented with reflective markers and strain gauges aimed at measuring their motion and their ground and cuff contact forces. They are considered as rigid bodies in the model, firmly connected to the hands of the subject and with no kinematic connection to his forearms.

The remaining of the paper is organized as follows: first, the experimental setup is described, including the motion capture, the measurement of ground reactions, and the active orthosis prototype. Then, the computational model used for the ID computation is described, including the introduction of the assistive devices, i.e. the crutches and the orthoses, and the proposed solution to the multiple-support problem. The last two Sections are devoted to the tests that have been carried out and the conclusions extracted from their results.

\section{EXPERIMENTAL SETUP \\ Motion Capture and Data Acquisition}

The gait laboratory is equipped with 12 OptiTrack Flex:V100 cameras. These cameras are capable of tracking reflective markers in a three-dimensional volume, at a $100 \mathrm{~Hz}$ frequency. The markers are placed on anatomical landmarks, thus allowing to reconstruct the motion of the subject. In addition, two AMTI AccuGait force plates are used to measure the foot-ground reactions, which are necessary for the ID calculation. These force plates measure the six components of the ground reaction, expressed as a wrench at the plate center. Since the OptiTrack cameras are low-cost equipment not intended for biomechanical applications, the included software does not allow to synchronize the optical motion capture to other devices, such as the aforementioned force plates. This is solved by using an in-house developed software, which takes advantage of the

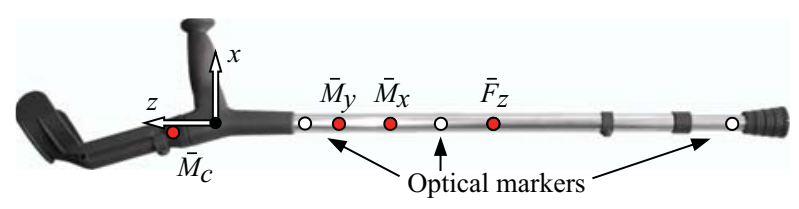

FIGURE 1. INSTRUMENTED CRUTCH DESCRIPTION.

TrackingTools $\mathrm{C}$ libraries provided by the manufacturer of the cameras.

The hardware synchronization is achieved by means of a National Instruments data acquisition system (DAQ), which acts as a master device, providing the synchronization signals to the force plates and the cameras. Moreover, it can itself simultaneously acquire any additional analog signal that may become necessary, such as EMG or, as in the present work, output voltages from extensometry devices.

\section{Instrumented Crutches}

SCI subjects are not able to walk without the help of supporting elements such as walkers, parallel bars or crutches. This means that the gait of SCI patients has in general more than two simultaneous ground contacts, as opposed to the case of biped gait, where the only interactions with the environment occur through the feet. Therefore, optical motion capture and force plates alone are no longer sufficient to calculate the joint torques.

When crutches are used as supporting elements, a possible solution could be the inclusion of more force plates, in order to measure the additional ground contact forces. However, it is difficult to ensure that the patient never sets a crutch and a foot on the same force plate, which would lead to an indeterminacy. Moreover, if the torques at the wrists were required, the forearmcuff interaction forces would still need to be measured, since they introduce additional unknowns in the ID problem. Therefore, the solution is to instrument the crutches themselves, in order to directly measure their cuff and ground contact forces.

These magnitudes can be measured, for instance, by placing six-axis transducers into the crutches [5]. However, due to the elevated cost or this solution, extensometry by means of strain gauge-based load cells has been chosen instead. Some simplifications have been assumed in order to reduce the number of measured magnitudes, i.e. the number of required Wheatstone bridges. On the one hand, since the crutch tips have a small contact area, the ground reaction is idealized as a force $\mathbf{F}_{t}$ acting at the tip center $\mathbf{r}_{t}$, thus neglecting the offset of the center of pressure. On the other hand, the cuff reaction is modeled as a normal force acting on its middle point. These simplifications reduce the number of unknowns from 12 to 4 per crutch, thus requiring a total of eight Wheatstone bridges, i.e. eight analog data acquisition channels.

Since the strain gauges measure the loads in local coordinates, the position and orientation of the crutches has to be de- 
termined by means of motion tracking. The placement of the optical markers can be seen in Fig. 1. Each crutch has the markers located at its outer side in the medio-lateral direction, being the upper and lower ones directly attached to the crutch body, and the middle ones mounted with an offset in the $y$ direction, in order to properly capture the crutch orientation.

As shown in Fig. 1, each load cell measures one magnitude. The lowest one measures the axial force $\bar{F}_{z}$, which is directly related to the axial component of the contact force $\bar{F}_{t z}$. The $\bar{M}_{x}$ and $\bar{M}_{y}$ cells measure the bending moments about the local $x$ and $y$ axes, which are mostly produced by the $\bar{F}_{t y}$ and $\bar{F}_{t x}$ components of $\mathbf{F}_{t}$, respectively. The remaining bridge captures also a bending moment $\bar{M}_{c}$ about the $y$ axis, but in this case at a point between the handle and the cuff, with the purpose of estimating the cuff contact force. Each one of the three lower load cells uses two $90^{\circ}$ rosettes in full-bridge configuration, whereas the upper ones are half bridges with two regular gauges each.

Crutches Calibration. The geometric and physical properties of the crutches, along with the strain gauge factors, could be used to analytically obtain the relationships between the measured magnitudes and the transducer voltages. However, this requires reliable knowledge of the material properties, and a high quality gauge attachment process, which is not always guaranteed. This problem can be overcome if the magnitude to be measured by the transducer is obtained simultaneously by other means, thus allowing to perform the calibration experimentally.

The cuff transducer is calibrated by clamping the crutch in horizontal position, with its local $y$ axis parallel to the ground, and loading its tip with different known weights, while noting the obtained voltages. The bending moment $\bar{M}_{c}$ is assumed to have a linear relationship with the output voltage $V_{c}$,

$$
\bar{M}_{c}=K_{c} V_{c}+\Delta_{c}
$$

where $K_{c}$ is the calibration constant and $\Delta_{c}$ is the DC offset. The different voltage-moment pairs $\left(V_{c}, \bar{M}_{c}\right)$ can be used to calculate the calibration parameters $K_{c}$ and $\Delta_{c}$ by performing a simple linear regression.

The remaining three load cells are calibrated simultaneously in order to account for possible cross-sensitivity problems. Numbering the load cells from bottom to top, the voltage-load relationships can be established as

$$
\left\{\begin{array}{l}
\bar{F}_{z} \\
\bar{M}_{x} \\
\bar{M}_{y}
\end{array}\right\}=\left[\begin{array}{lll}
K_{11} & K_{12} & K_{13} \\
K_{21} & K_{22} & K_{23} \\
K_{31} & K_{32} & K_{33}
\end{array}\right]\left\{\begin{array}{l}
V_{1} \\
V_{2} \\
V_{3}
\end{array}\right\}+\left\{\begin{array}{l}
\Delta_{1} \\
\Delta_{2} \\
\Delta_{3}
\end{array}\right\}
$$

The calibration process consists of pressing the crutch tip against a force plate, trying to produce axial and bending loads, while si- multaneously capturing its motion. The ground reaction $\mathbf{F}_{t}$ measured by the plate, along with the inertia and gravity forces derived from the motion capture data, can be used to calculate the transducer loads by stating the Newton-Euler equations for the portion of the crutch between each load cell and the ground

$$
\begin{aligned}
\bar{F}_{z} & =\mathbf{w}^{\top}\left[m_{3}\left(\ddot{\mathbf{r}}_{g 3}-\mathbf{g}\right)-\mathbf{F}_{t}\right] \\
\bar{M}_{x} & =\bar{I}_{2}\left(\bar{\alpha}_{x}-\bar{\omega}_{y} \bar{\omega}_{z}\right) \\
& -\mathbf{u}^{\top}\left[\left(\mathbf{r}_{t}-\mathbf{r}_{2}\right) \times \mathbf{F}_{t}+m_{2}\left(\mathbf{r}_{2}-\mathbf{r}_{g 2}\right) \times\left(\ddot{\mathbf{r}}_{g 2}-\mathbf{g}\right)\right] \\
\bar{M}_{y} & =\bar{I}_{1}\left(\bar{\alpha}_{y}+\bar{\omega}_{z} \bar{\omega}_{x}\right) \\
& -\mathbf{v}^{\top}\left[\left(\mathbf{r}_{t}-\mathbf{r}_{1}\right) \times \mathbf{F}_{t}+m_{1}\left(\mathbf{r}_{1}-\mathbf{r}_{g 1}\right) \times\left(\ddot{\mathbf{r}}_{g 1}-\mathbf{g}\right)\right]
\end{aligned}
$$

The three unit vectors $\mathbf{u}, \mathbf{v}$ and $\mathbf{w}$ define the local axes of the crutch, expressed in global coordinates; $\bar{\omega}$ and $\bar{\alpha}$ are the angular velocity and acceleration of the crutch in local coordinates, and $\mathbf{g}$ is the acceleration of gravity; $\mathbf{r}_{i}$ is the absolute location of load cell $i ; m_{i}$ and $\overline{I_{i}}$ represent the mass and moment of inertia in local coordinates of the portion of the crutch between $\mathbf{r}_{i}$ and $\mathbf{r}_{t}$, about its center of gravity $\mathbf{r}_{g i}$. These segments are considered axisymmetric, in such a way that $\bar{I}_{x i}=\bar{I}_{y \underline{i}}=\bar{I}_{i}$, being their moment of inertia about the longitudinal axis $I_{z i}$ neglected.

If a crutch is pressed against a plate for a time period $t$, there will be $100 t$ data points available, since the motion capture system uses a $100 \mathrm{~Hz}$ sampling rate. These points include the transducer calibration loads $\bar{F}_{z}, \bar{M}_{x}$ and $\bar{M}_{y}$, and their voltage counterparts $V_{1}, V_{2}$ and $V_{3}$. Eqn. 2 should be fulfilled at all of them, so that a standard least-squares minimization procedure can be used in order to calculate the optimal $K_{i j}$ and $\Delta_{i}$ calibration parameters. The results obtained with the actual crutches when applying the described procedure have a coefficient of determination $R^{2}$ above 0.95 , reaching values higher than 0.99 in the axial load cells, thus indicating that the load cells have a good linearity.

\section{Active Knee-Ankle-Foot Orthoses}

The orthosis design is shown in Fig. 2. It consists of a standard leg brace like those commonly used by SCI patients, with some modifications aimed at allowing to control the knee swing.

The knee articulation of the leg brace has been substituted by two devices: a locking device in the inner joint, manufactured by Fior \& Gentz, and a Maxon EC-45 flat motor in the outer joint, equipped with an encoder and a 1:156 gear. The objective of the locking device is to avoid using the motor for locking the knee during stance, thus reducing the power consumption of the orthosis. The ankle articulation has been substituted by a foot plate with a Klenzack spring that prevents the drop-foot, and another encoder to measure the ankle plantar and dorsiflexion angle. Additionally, pressure sensors are placed on the foot plate in order to detect when the foot is in contact with the ground. In what respects the optical motion capture, the knee and ankle markers are 


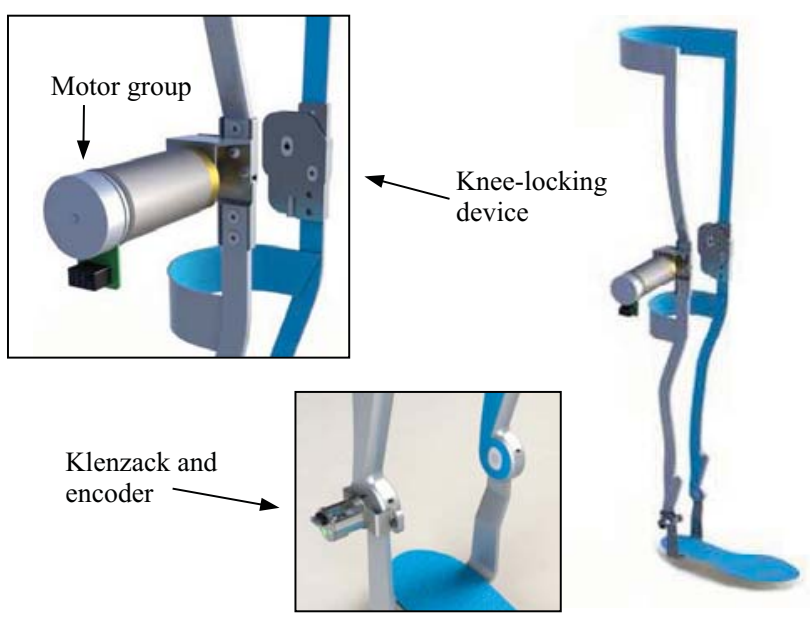

FIGURE 2. ACTIVE KNEE-ANKLE-FOOT ORTHOSIS.

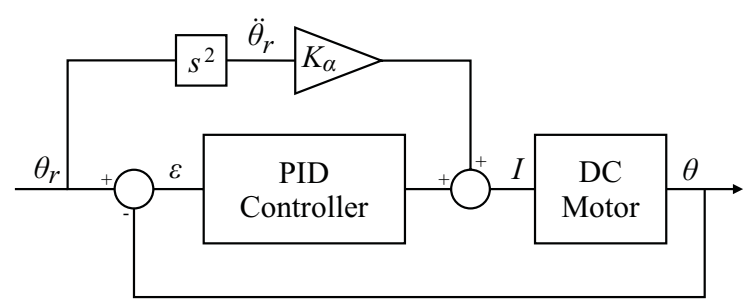

FIGURE 3. BLOCK DIAGRAM OF THE MOTOR CONTROLLER.

now placed on the orthosis instead of directly on the subject, thus requiring a modification of the motion reconstruction equations.

The motors at the knees are controlled by the commercial EPOS2 interfaces provided by Maxon, whose position control mode uses a modified PID controller with acceleration feedforward. The transfer function of the PID controller is the following:

$$
G(s)=\frac{I(s)}{\varepsilon(s)}=K_{P}+\frac{K_{I}}{s}+\frac{K_{D} s}{1+\frac{K_{D}}{16 K_{P}} s}
$$

where $K_{P}, K_{I}$ and $K_{D}$ are the proportional, integral and derivative gains, respectively, $I$ is the motor input current, and $\varepsilon$ is the difference between the required angle at the knee $\theta_{r}$, and the actual angle $\theta$ measured by the knee encoder. The information provided by all the sensors can be used in many ways to determine the required knee angle, thus leading to different control schemes. A block diagram of the complete loop is shown in Fig. 3, where the DC Motor block represents the whole dynamics of the motororthosis-subject system.

\section{COMPUTATIONAL MODEL}

A 3D computational model of the subject has been developed in mixed (natural + angular) coordinates [7]. The model, shown in Fig. 4b possesses 18 bodies and 57 degrees of freedom, and it is defined by 228 dependent coordinates. All the body segments are connected by spherical joints in the model, so as to circumvent the problem of determining the rotation axes. Each foot is defined by means of two segments. The global axes are defined as follows: $x$ axis in the antero-posterior direction, $y$ axis in the medio-lateral direction, and $z$ axis in the vertical direction.

Following the picture in Fig. 4b, the subset of natural coordinates, $\mathbf{q}$, comprises the three Cartesian coordinates of 22 points and the three Cartesian components of 36 unit vectors, thus making a total of 174 variables. The points correspond to the positions of all the spherical joints, along with the centers of mass of the five distal segments (head, hands and forefeet). Each one of the 18 bodies is defined by its proximal and distal points, plus two orthogonal unit vectors aligned at the antero-posterior and medio-lateral directions, respectively, when the model is in a standing posture. The remaining 54 variables, which are used as part of a subset $\mathbf{z}$ of independent coordinates, are the 18 sets of 3 angles that define the orientation of each body with respect to the inertial frame.

The geometric parameters of the model are obtained, for the lower limbs, by applying correlation equations from a reduced set of measurements taken on the subject [8] and, for the upper part of the body, by scaling table data according to the mass and height of the subject $[9,10]$. Regarding the inertial parameters, they are obtained, for the lower limbs, by a correction, based on data coming from densitometry (DXA) if available, of the method already indicated for the geometric parameters; for the upper part of the body, the scaling method is used again, but a second scaling is applied in order to adjust the total mass of the subject.

The position histories of the markers are used to calculate the histories of the model natural coordinates $\mathbf{q}^{*}$ by means of simple algebraic relations [8]. The values of these coordinates at each instant of time are not kinematically consistent due to the inherent errors of the motion capture process and to the fact that the joints are not ideal [10-13]. Therefore, the kinematic consistency of the natural coordinates at position level is imposed, at each instant of time, by solving the following minimization problem:

$$
\begin{array}{ll}
\underset{\mathbf{q}}{\operatorname{minimize}} & f(\mathbf{q})=\left(\mathbf{q}-\mathbf{q}^{*}\right)^{\top} \mathbf{W}\left(\mathbf{q}-\mathbf{q}^{*}\right) \\
\text { subject to } & \Phi(\mathbf{q})=0
\end{array}
$$

where $\mathbf{W}$ is a weighting matrix that allows to assign different weights to the different coordinates according to their expected errors, and $\Phi$ is the vector of kinematic constraint equations. 

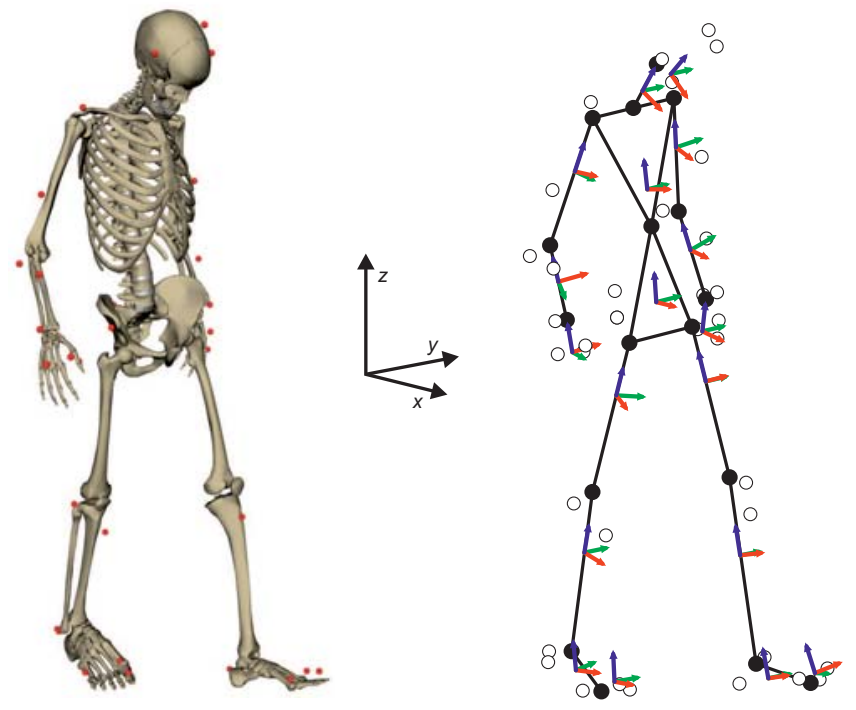

FIGURE 4. (A) LOCATION OF MARKERS (B) COMPUTATIONAL MODEL

This problem can be iteratively solved by means of the following augmented Lagrangian minimization process [11],

$$
\begin{aligned}
\left(\mathbf{W}+\Phi_{\mathbf{q}}^{\top} \kappa \Phi_{\mathbf{q}}\right) \Delta \mathbf{q}_{i+i} & =-\mathbf{W}\left(\mathbf{q}_{i}-\mathbf{q}^{*}\right)-\Phi_{\mathbf{q}}^{\top}\left(\kappa \Phi+\lambda_{i}\right) \\
\lambda_{i+1} & =\lambda_{i}+\kappa \Phi \quad i=1,2, \ldots
\end{aligned}
$$

where $\Delta \mathbf{q}_{i+i}=\mathbf{q}_{i+1}-\mathbf{q}_{i}, \Phi_{\mathbf{q}}$ is the Jacobian matrix of the kinematic constraints vector $\Phi, \lambda$ is the vector of Lagrange multipliers, and $\kappa$ is the penalty factor.

From the consistent values of the natural coordinates $\mathbf{q}$, a set of independent coordinates $\mathbf{z}$ is calculated. This set is composed of the three Cartesian coordinates of the lumbar joint (spherical joint connecting pelvis and torso, see Fig. 4b), along with the three $x, y, z$ rotation angles of the 18 bodies with respect to the fixed global axes, thus defining the total 57 degrees of freedom. Prior to differentiate the histories of the independent coordinates $\mathbf{z}$, the SSA filter [14] is applied to them in order to reduce the noise introduced by the motion capture and the kinematic consistency. Then, the Newmark's integrator expressions are used to numerically differentiate the filtered position histories so as to obtain the corresponding velocity $\dot{\mathbf{z}}$ and acceleration $\ddot{\mathbf{z}}$ histories [11], thus completing the description of the motion.

Once the histories of the independent coordinates $\mathbf{z}$, and their derivatives, $\dot{\mathbf{z}}$ and $\ddot{\mathbf{z}}$, have been obtained, the ID problem is solved by means of the velocity transformation formulation known as Matrix-R [7], which provides the motor efforts required to generate the motion, in the form of generalized forces applied to the independent coordinates $\mathbf{z}$. The formulation is based on a velocity transformation matrix $\mathbf{R}$ such that $\dot{\mathbf{q}}=\mathbf{R} \dot{\mathbf{z}}$. If this transformation is applied to the Lagrange's equations of motion of the first kind, then the equations of motion in independent or minimum number of coordinates are obtained as:

$$
\mathbf{R}^{\top} \overline{\mathbf{M}} \mathbf{R} \ddot{\mathbf{z}}=\mathbf{R}^{\top}(\overline{\mathbf{Q}}-\overline{\mathbf{M}} \dot{\mathbf{R}} \dot{\mathbf{z}})+\mathbf{Q}_{m}
$$

where $\overline{\mathbf{M}}$ and $\overline{\mathbf{Q}}$ are the mass matrix and the generalized forces vector (excluding ground interactions and joint torques) expressed in the natural coordinates $\mathbf{q}$, and $\mathbf{Q}_{m}$ are the motor efforts applied to the independent coordinates, i.e. the unknowns of the ID problem.

The ID problem can be solved in order to obtain the forces that have been or need to be applied to achieve a determined motion. In general, the number of forces and torques required for producing a given motion is equal to the number of degrees of freedom of the system. Therefore, solving an ID problem for the described model can only obtain the six components of the net external forces and moments acting on the system. In the present work, the six degrees of freedom chosen to connect the model to the environment are the three rotation angles of the pelvis with respect to the inertial system, and the three components of the position of the lumbar joint, i.e. that connecting the pelvis to the torso. It is obvious that the intersegmental torques obtained from this ID problem are not the actual torques produced during the motion, but, provided that independent measurements of the ground reactions are available, they can be used as a verification of the accuracy of the ID problem.

\section{Introduction of Crutches in the Model}

The crutches are introduced in the model as rigid bodies, in natural coordinates as the rest of the model. They are modeled using two points: the intersection between the crutch bar and the handle, which is the origin, and the tip. Two orthogonal unit vectors, one of them pointing forward and another one orthogonal to the first one and to the crutch axis, are required to complete the modeling, thus adding a total of 24 variables to the model (4 points and 4 vectors). The crutches do not add any degree of freedom to the system, since they are considered as clamped to the hands. Therefore, the wrist rotation angles are shared between the crutches and the hands.

The contact forces, as explained when describing the experimental setup, are considered as acting at the tip of the crutch. By applying Eqn. (2) to the voltages of the Wheatstone bridges, the transducer loads are obtained. Then, the equilibrium equations used for the calibration are applied in the reverse way to calculate the contact forces $\mathbf{F}_{t}$. After some manipulations, they can be 
expressed in the form of a $3 \times 3$ linear system:

$$
\begin{gathered}
{\left[\begin{array}{c}
\mathbf{w}^{\top} \\
\left(\mathbf{r}_{2}-\mathbf{r}_{t}\right)^{\top} \tilde{\mathbf{u}} \\
\left(\mathbf{r}_{1}-\mathbf{r}_{t}\right)^{\top} \tilde{\mathbf{v}}
\end{array}\right]\left\{\begin{array}{l}
F_{t x} \\
F_{t y} \\
F_{t z}
\end{array}\right\}=} \\
\left\{\begin{array}{c}
m_{3} \mathbf{w}^{\top}\left(\ddot{\mathbf{r}}_{g 3}-\mathbf{g}\right)-\bar{F}_{z} \\
\overline{I_{2}}\left(\bar{\alpha}_{x}-\bar{\omega}_{y} \bar{\omega}_{z}\right)-\mathbf{u}^{\top}\left[m_{2}\left(\mathbf{r}_{2}-\mathbf{r}_{g 2}\right) \times\left(\ddot{\mathbf{r}}_{g 2}-\mathbf{g}\right)\right]-\bar{M}_{x} \\
\overline{I_{1}}\left(\bar{\alpha}_{y}+\bar{\omega}_{z} \bar{\omega}_{x}\right)-\mathbf{v}^{\top}\left[m_{1}\left(\mathbf{r}_{1}-\mathbf{r}_{g 1}\right) \times\left(\ddot{\mathbf{r}}_{g 1}-\mathbf{g}\right)\right]-\bar{M}_{y}
\end{array}\right\}
\end{gathered}
$$

\section{Introduction of Orthoses in the Model}

The orthoses are introduced in the model by simply altering the inertia properties of the thighs, calves and ankles accordingly.

The Klenzack spring is introduced in the model as a torque $\tau_{a}$ along the ankle articulation axis, whose characteristics have been experimentally measured:

$$
\tau_{a}=5.563-11.104 \theta_{a} \quad(\mathrm{Nm})
$$

$\theta_{a}$ is the ankle angle in radians, considered to be zero in the neutral standing position, and positive in the direction of plantar flexion. The Klenzack articulation allows the angle to vary between 0 and 20 degrees.

In order to introduce the swing control in the numerical simulation, the transfer function of the controller (i.e. the PID described in Eqn. (6) and the feed-forward) needs to be appropriately discretized. This is achieved by first applying the inverse Laplace transform to the transfer function. Then, the time derivatives are substituted by backward differences, and the time integrals by finite sums. This leads to a discrete form of the controller that can be used to obtain the motor torque at time-step $k$ as follows:

$$
\tau_{k}=K_{1} \tau_{k-1}+K_{2} \varepsilon_{k}+K_{3} \frac{\varepsilon_{k}-\varepsilon_{k-1}}{h}+K_{4} h \sum_{i=1}^{k} \varepsilon_{i}+K_{5} \ddot{\theta}_{r}
$$

where $h$ is the time-step length used for the numerical simulation. The five constants $K_{1}$ to $K_{5}$ are defined as:

$$
\begin{aligned}
& K_{1}=K_{D} / k \\
& K_{2}=\left(16 K_{P}^{2}+K_{D} K_{I}\right) K_{m} h / k \\
& K_{3}=17 K_{P} K_{D} K_{m} h / k \\
& K_{4}=16 K_{P} K_{I} K_{m} h / k \\
& K_{5}=K_{\alpha} K_{m}
\end{aligned}
$$

where the constant $k$ is equal to $\left(16 K_{P} h+K_{D}\right), K_{\alpha}$ is the gain of the acceleration feed-forward shown in Fig. 3, which is intended to provide extra torque when high accelerations are required, and $K_{m}$ is the factor used to convert currents into torques, including the torque constant of the motor and the gear ratio (1:156).

The torques obtained from the controller and the Klenzack springs can be directly introduced in the system as part of the known generalized forces $\overline{\mathbf{Q}}$ of Eqn. (9), in such a way that the ID will yield the remaining unknown torques in $\mathbf{Q}_{m}$, i.e. those provided by the subject.

\section{Multiple-Support Problem in Inverse Dynamics}

The generalized forces $\mathbf{Q}_{m}$ obtained from the inverse dynamic process described above do not correspond to the actual external reactions and internal motor torques, since the independent coordinates $\mathbf{z}$ are the position of the lumbar joint and the orientation angles of all the bodies. In order to calculate the actual motor torques, the generalized forces corresponding to the angles need to be transformed into torques, and the ground reactions must be properly shared between the contacting feet and crutches.

The first step for calculating the actual reactions and torques consists of transforming the generalized forces $\mathbf{Q}_{m}$ into an equivalent set of internal torques $\mathbf{T}^{*}$ and external reaction forces $\mathbf{F}^{*}$ and moments $\mathbf{M}^{*}$, where the six components of the external reactions are considered as acting on the pelvis, at the lumbar joint. The three components of $\mathbf{F}^{*}$ are directly obtained as the generalized forces associated to the position of the lumbar joint, and $\mathbf{M}^{*}$ is calculated by applying the virtual power principle to the generalized forces corresponding to the pelvis angles.

Then, the external reactions from ID are translated from the lumbar joint to its projection into the ground $\mathbf{r}_{0}$, and the same is done for those measured at the feet and crutches. If the contacting elements (right foot, left foot, right crutch and left crutch) are numbered from 1 to 4 , their measured reactions can be denoted as $\mathbf{F}_{j}^{s}$ and $\mathbf{M}_{j}^{s}$. At this point, the reactions from both ID and sensor outputs are located at a common point, in such a way that the moments can be compared. Due to the large number of error sources present in the process, the sum of the four measured reactions is not exactly equal to the net reactions obtained from ID, being the discrepancy among them an indicator of the global accuracy of the process.

The next step consists of estimating the amount of the ID reactions that corresponds to each contacting element. There exist several methods to combine the results from ID with the measured reactions in a least-squares sense $[15,16]$, but they yield torques that are not fully consistent with the measured motion. In order to obtain fully consistent results, a simpler alternative method is presented here.

The total reaction calculated from ID must be shared among all contacting elements, but keeping the resultant consistent with the ID. This is achieved by splitting the residual between the ID and measured reactions among the contacting elements. In or- 
der to avoid discontinuities and to minimize relative errors, the amount of error added to each sensor is proportional to its relative magnitude. Since the crutches have only force measurements, and their center of pressure is determined by the motion, forces and moments use a different sharing strategy.

The residual in the three force components is

$$
\varepsilon_{F}=\mathbf{F}^{*}-\sum_{j=1}^{4} \mathbf{F}_{j}^{s}
$$

and the amount of error corresponding to the $i^{\text {th }}$ component of the force acting at element $j$ can be obtained as

$$
k_{i j}=\frac{\left|F_{i j}^{s}\right|}{\sum_{j=1}^{4}\left|F_{i j}^{s}\right|} \quad j=1,2,3,4
$$

Therefore, the component $i$ of the corrected reaction force at element $j$ remains

$$
F_{i j}=F_{i j}^{*}+k_{i j} \varepsilon_{F i} \quad j=1,2,3,4
$$

thus ensuring that the resultant of the four shared reaction forces is equal to $\mathbf{F}^{*}$. Once the forces have been calculated, the next step is to use the crutch forces and the location of their contact points to obtain their already corrected moments $\mathbf{M}_{3}$ and $\mathbf{M}_{4}$. This leaves the feet moments $\mathbf{M}_{1}$ and $\mathbf{M}_{2}$ to be corrected. The residual in this case is

$$
\varepsilon_{M}=\mathbf{M}^{*}-\sum_{j=1}^{2} \mathbf{M}_{j}^{S}-\sum_{j=3}^{4} \mathbf{M}_{j}
$$

and the sharing factors are obtained as

$$
k_{i j}=\frac{\left|M_{i j}^{s}\right|}{\sum_{j=1}^{2}\left|M_{i j}^{s}\right|} \quad j=1,2
$$

so that the corrected moments can be finally calculated

$$
M_{i j}=M_{i j}^{*}+k_{i j} \varepsilon_{M i} \quad j=1,2
$$

Once the full set of reaction forces $\mathbf{F}$ and moments $\mathbf{M}$ whose resultants are consistent with the ID is available, they are translated back from $\mathbf{r}_{0}$ to their actual locations (feet and crutch tips), and the virtual power principle is then used to redistribute the internal torques corresponding to the new position of the external

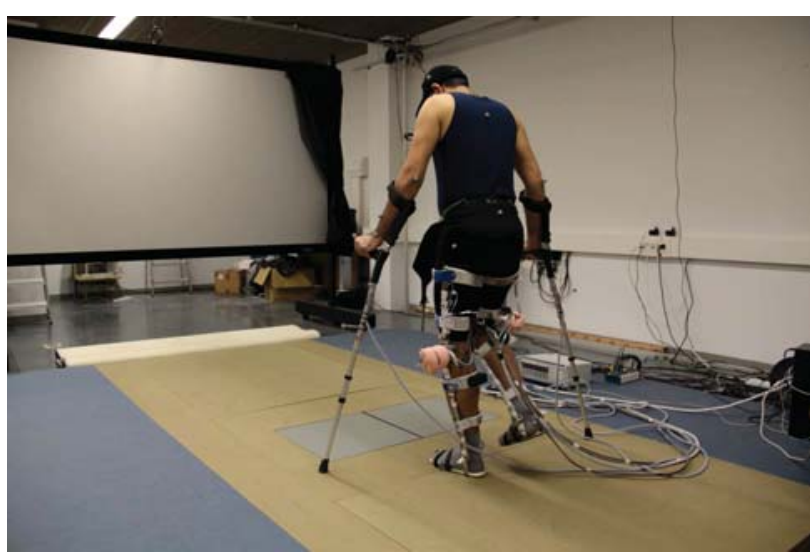

FIGURE 5. MOTION CAPTURE PROCESS.

interactions. The whole process leads to motor torques and reactions that are consistent with the ID, and have the reaction forces shared in the same proportions as the measured ones. The sensor information, rather than an input to the ID problem, is both a reference for validating the ID results, and a sharing criterion for the multiple support problem.

\section{TESTS AND RESULTS}

The experimental tests have been carried out by a healthy male subject, with a height of $1.60 \mathrm{~m}$ and a weight of $74 \mathrm{Kg}$. The subject is wearing a pair of active orthoses, as seen in Fig. 5, while walking over two force plates with the help of the instrumented crutches. The motion of the reflective markers attached to the subject and the crutches is captured by the IR cameras, in full synchronization with all the sensors. The orthoses are controlled by using a simple scheme: each knee angle follows a fixed time history, similar to that of normal gait, as it loses contact with the ground. The shape of the reference time history is scaled in time, so as to be adapted to the gait speed.

In Fig. 6, the comparison among the net reaction forces and moments from the sensors and the ID is shown. It can be seen that the net reactions obtained by using only motion and inertial data (ID) are in good correlation with their measured counterparts, considering the errors introduced by the BSP estimation, the motion capture, etc.

The reactions at the feet and crutches, obtained after applying the sharing technique described above to the ID results from Fig. 6, are displayed in Figs. 7 and 8. The plots are restricted to the time when feet are completely inside the force plates, since the results obtained with a foot totally or partially out of a plate are not valid. The moments are considered in both Figures with respect to the projection of the lumbar joint into the ground, i.e. the already mentioned $\mathbf{r}_{0}$ point.

In Fig. 9, the flexion-extension torques at the lower joints 

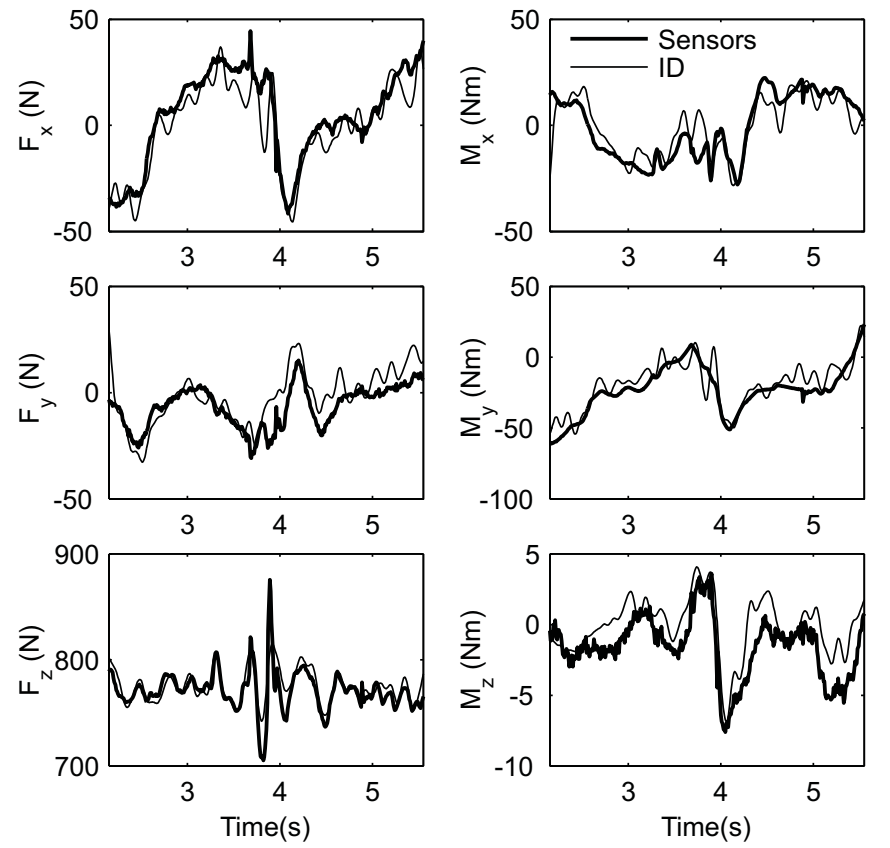

FIGURE 6. NET GROUND REACTIONS: INVERSE DYNAMICS VS. MEASUREMENTS.

are shown. These torques are only those applied by the subject, since the orthosis and Klenzack torques are introduced during the ID as known generalized forces.

\section{CONCLUSIONS}

In this work, a procedure to perform the ID analysis of a subject walking with the aid of active orthoses and crutches is presented. A full experimental setup has been established, including motion capture, reactions measurement, and active orthoses. The measured data is fed into a computational model that allows to solve the ID problem considering the effect of all the assistive devices, including the simulation of the motor control.

The analysis system has the ability of verifying the quality of the measurements, since the ground reaction forces are simultaneously obtained from two different sources, and therefore they can be compared. The results obtained in the tests show a reasonably good accuracy, even though the system is built on low-cost equipment, not designed for biomechanical applications.

The ID analysis of the gait can be a great help in the performance assessment of active orthoses, as well as for control design, since it must be completely customized to fit each individual patient. It can also be used for evaluating the possible advantages of active orthoses over passive ones, for monitoring the adaptation of the patient, and for improving the understanding of the patient-orthosis interaction.

This work is part of a project aimed at developing a pre-
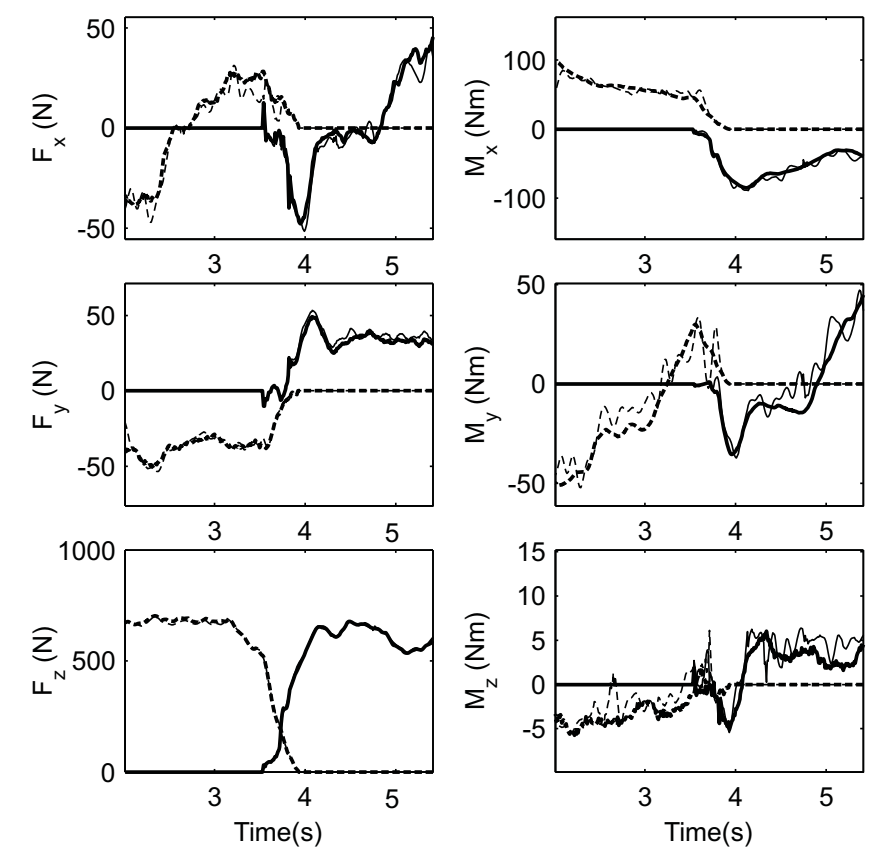

FIGURE 7. GROUND REACTIONS (SOLID: RIGHT FOOT, DOTTED: LEFT FOOT, THICK: FORCE PLATES, THIN: ID).

dictive dynamics-based tool $[17,18]$ for the design of active orthoses, which would allow to test assistive devices in virtual conditions. This would reduce the number of required tests, thus improving the design cycle of the orthosis.

\section{ACKNOWLEDGMENT}

The support of this work by the Spanish Ministry of Science and Innovation under the project DPI2009-13438-C03, cofinanced by the European Union through EFRD funds, is acknowledged.

\section{REFERENCES}

[1] Dollar, A. M., and Herr, H., 2008. "Lower extremity exoskeletons and active orthoses: Challenges and state-ofthe-art". IEEE Transactions on Robotics, 24(1), pp. 144158.

[2] Yakimovich, T., Lemaire, E., and Kofman, J., 2009. “Engineering design review of stance-control knee-ankle-foot orthoses.". Journal of rehabilitation research and development, 46(2), p. 257.

[3] Lugrís, U., Carlín, J., Michaud, F., and Cuadrado, J., 2012. "Joint efforts calculation in the gait of incomplete spinal cord injured subjects". In Book of Abstracts of the Second Joint International Conference on Multibody System Dynamics - IMSD 2012, pp. 72-73. 

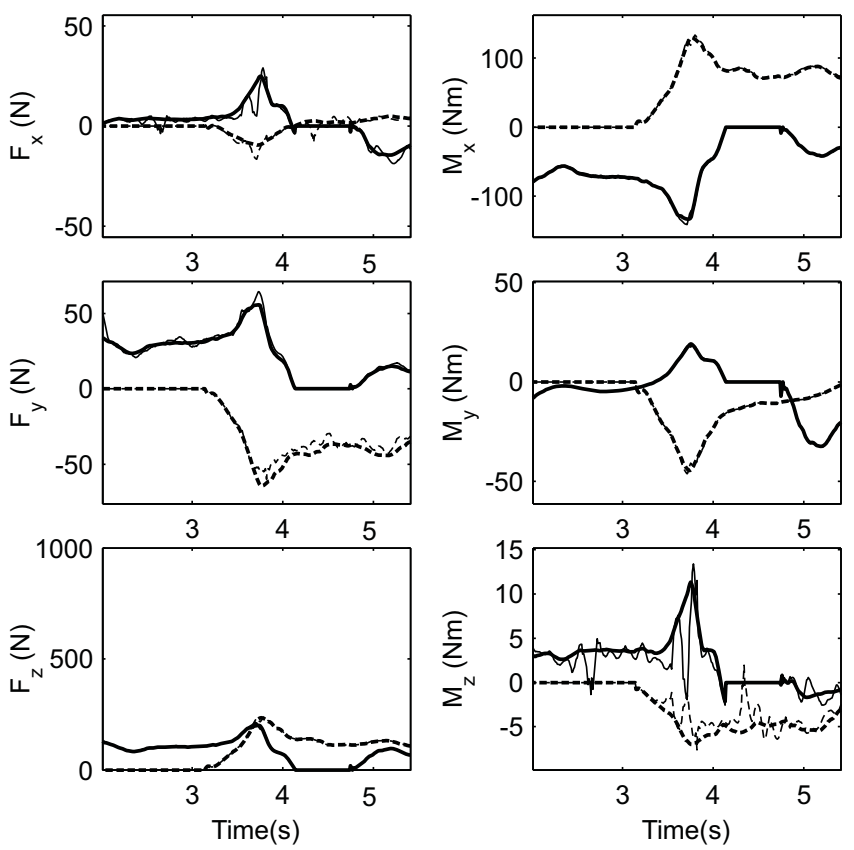

FIGURE 8. GROUND REACTIONS (SOLID: RIGHT CRUTCH, DOTTED: LEFT CRUTCH, THICK: WHEATSTONE BRIDGES, THIN: ID).
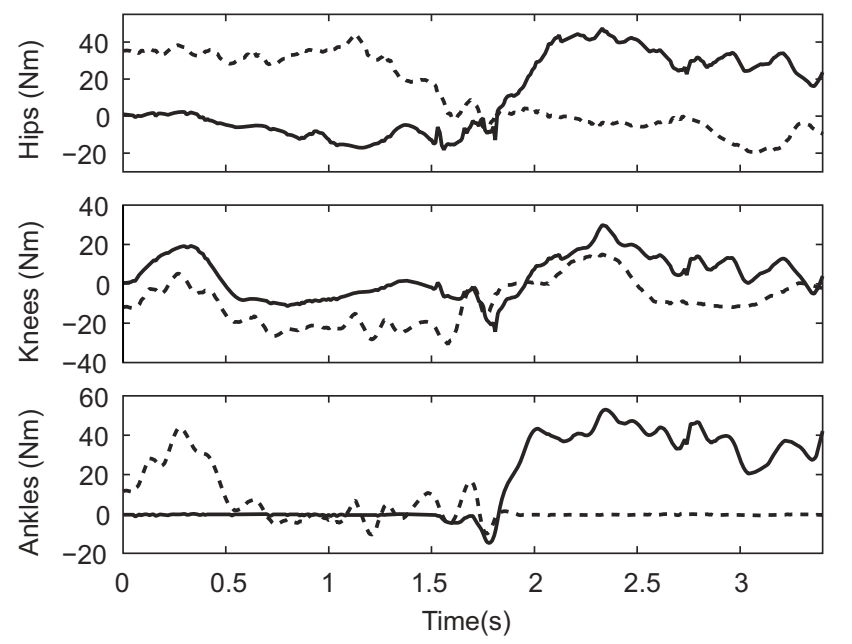

FIGURE 9. FLEXION-EXTENSION TORQUES (SOLID: RIGHT LEG, DOTTED: LEFT LEG).

[4] Slavens, B., Sturm, P., and Harris, G., 2010. "Upper extremity inverse dynamics model for crutch-assisted gait assessment". Journal of Biomechanics, 43(10), pp. 20262031.

[5] Slavens, B., Bhagchandani, N., Wang, M., Smith, P., and Harris, G., 2011. "An upper extremity inverse dynamics model for pediatric Lofstrand crutch-assisted gait". Journal of Biomechanics, 44(11), pp. 2162-2167.

[6] Font-Llagunes, J. M., Pàmies-Vilà, R., Alonso, J., and Lugrís, U., 2011. "Simulation and design of an active orthosis for an incomplete spinal cord injured subject". Procedia IUTAM, 2(0), pp. 68-81.

[7] García de Jalón, J., and Bayo, E., 1994. Kinematic and Dynamic Simulation of Multibody Systems: The Real-Time Challenge. Springer-Verlag, Berlin.

[8] Vaughan, C. L., Davis, B. L., and O'Connor, J. C., 1999. Dynamics of Human Gait, 2nd Edition. Kiboho Publishers, Cape Town.

[9] Rodrigo, S., Ambrósio, J., Da Silva, M., and Penisi, O., 2008. "Analysis of human gait based on multibody formulations and optimization tools". Mechanics Based Design of Structures and Machines, 36(4), pp. 446-477.

[10] Silva, M., and Ambrósio, J., 2002. "Kinematic data consistency in the inverse dynamic analysis of biomechanical systems". Multibody System Dynamics, 8(2), pp. 219-239.

[11] Alonso, F., Cuadrado, J., Lugrís, U., and Pintado, P., 2010. "A compact smoothing-differentiation and projection approach for the kinematic data consistency of biomechanical systems". Multibody System Dynamics, 24(1), pp. 67-80.

[12] Lu, T. W., and O'Connor, J. J., 1999. "Bone position estimation from skin marker co-ordinates using global optimisation with joint constraints". Journal of Biomechanics, 32(2), pp. 129-134.

[13] Reinbolt, J. A., Schutte, J. F., Fregly, B. J., Koh, B. I., Haftka, R. T., George, A. D., and Mitchell, K. H., 2005. "Determination of patient-specific multi-joint kinematic models through two-level optimization". Journal of Biomechanics, 38(3), pp. 621-626.

[14] Golyandina, N., Nekrutkin, V., and Zhigljavsky, A., 2001. Analysis of Time Series Structure: SSA and Related Techniques. Chapman \& Hall/CRC.

[15] van den Bogert, A. J., and Su, A., 2008. "A weighted least squares method for inverse dynamic analysis". Computer Methods in Biomechanics and Biomedical Engineering, 11(1), pp. 3-9.

[16] Kuo, A., 1998. "A least-squares estimation approach to improving the precision of inverse dynamics computations". Journal of Biomechanical Engineering, 120(1), pp. 148159.

[17] Ren, L., Jones, R. K., and Howard, D., 2007. "Predictive modelling of human walking over a complete gait cycle". Journal of Biomechanics, 40(7), pp. 1567-1574.

[18] Xiang, Y., Chung, H. J., Kim, J. H., Bhatt, R., Rahmatalla, S., Yang, J., Marler, T., Arora, J., and Abdel-Malek, K., 2010. "Predictive dynamics: An optimization-based novel approach for human motion simulation". Structural and Multidisciplinary Optimization, 41(3), pp. 465-479. 